\title{
EVALUATION OF VARIOUS METHODS OF TROCHANTERIC FRACTURE FIXATION AND THEIR COMPARISON- A PROSPECTIVE STUDY
}

\author{
Gajraj Singh1, Sandhya Gautam², Naved Ahmed³, Chhaya Garg4, Sunil Kumar 5
}

${ }_{1}^{1}$ Associate Professor, Department of Orthopaedics, LLRM Medical College, Meerut, Uttar Pradesh, India.

${ }^{2}$ Associate Professor, Department of Medicine, LLRM Medical College, Meerut, Uttar Pradesh, India.

$3 J u n i o r$ Resident, Department of Orthopaedics, LLRM Medical College, Meerut, Uttar Pradesh, India.

${ }_{4}^{4}$ Assistant Professor, Department of SPM (Social \& Preventing Medicine) LLRM Medical College, Meerut, Uttar Pradesh, India. 5 Junior Resident, Department of Orthopaedics, LLRM Medical College, Meerut, Uttar Pradesh, India.

\section{ABSTRACT}

\section{BACKGROUND}

Trochanteric fractures are devastating injuries that most commonly affect the elderly and also the young; they have tremendous impact on both the health care system, and society in general. ${ }^{1}$ The mainstay of the treatment of intertrochanteric fractures is fixation with DHS, intramedullary device, and proximal locking compression plate. The aim of the present study was to compare the result in terms of rate of union, time of ambulation and functional recovery of fracture intertrochanteric femur treated by either dynamic hip screw (DHS), proximal femoral interlocking nail (PFN) or proximal locking compression plate (PFLCP).

\section{METHODS}

This was a prospective study of 62 patients, 20 patients were treated by PFN (Group A), 32 cases were treated by DHS (Group B) and 10 cases treated by PFLCP (Group C). All Patients were followed up at 1 month, 3 months, 6 months and 1 year. Results were compared for functional outcome by using Harris Hip Score.

\section{RESULTS}

Comparison of Harris hip score at 12-month-follow-up period revealed that PFN group to be significantly more mobile (PFN- 94.8 vs. DHS- 90.8 vs. PFLCP- 89.3). p-Value for PFN vs. DHS group is 0.12 , p value for PFN vs. PFLCP is $0.073(>0.05)$ and p value for DHS vs. PFLCP is 1.00. All p values show insignificant difference in functional outcome of patients. It shows that most of patients achieved approximately same level of functional outcome at the end of one year.

\section{CONCLUSIONS}

PFN is a biomechanically good device for fixation of comminuted intertrochanteric fracture femur. PFN allows faster mobilization, early rehabilitation, lower implant failure rate, and faster union as compared to DHS and PFLCP. In our study, PFLCP seems to be feasible alternative to PFN and DHS in certain complex, unstable, comminuted trochanteric fractures when weight bearing was restricted till radiological signs of fractures healing are seen.

HOW TO CITE THIS ARTICLE: Singh G, Gautam S, Ahmed N, et al. Evaluation of various methods of trochanteric fracture fixation and their comparison-a prospective study. J. Evolution Med. Dent. Sci. 2019;8(17):1388-1393, DOI: 10.14260/jemds/2019/309

\section{BACKGROUND}

Intertrochanteric fractures are one of the most common fracture of the hip especially in the elderly with osteoporotic bones, usually due to low-energy trauma like simple falls. ${ }^{2}$ The incidence of intertrochanteric fractures varies from country to country. Gulberg et $\mathrm{al}^{3}$ has predicted that the total number of hip fractures will reach 2.6 million by 2025 and 4.5 million by 2050 . The treatment of choice in fracture intertrochanteric femur is internal fixation. There are various forms of internal fixation devices used for trochanteric fractures i.e. DHS, PFN, PFLCP

The most commonly used device is the dynamic hip screw with slide plate assemblies. Dynamic Hip Screw (DHS) is still considered the gold standard for treating intertrochanteric fractures by many.

'Financial or Other Competing Interest': None.

Submission 02-02-2018, Peer Review 22-02-2019,

Acceptance 28-02-2019, Published 29-04-2019.

Corresponding Author:

Dr. Gajraj Singh,

R-21, Campus LLRM Medical College,

Meerut-250004,

Uttar Pradesh

India.

E-mail: gajrajsingh179@gmail.com

DOI: $10.14260 /$ jemds $/ 2019 / 309$
The advantages and disadvantages of the DHS have been well established in several studies done in the past, 4 this is a collapsible fixation device, which permits the proximal fragment to collapse or settle on the fixation device, seeking its own position of stability. Stable fractures can be very well treated with dynamic hip screw alone with good results proven by various studies. It is the unstable fractures which are difficult to manage with dynamic hip screw alone. Rates of complications like screw cut out, shortening of limb, varus deformity of proximal femur, and even non-union are higher in unstable fractures as compared with stable fractures.

Arbeitsegmenin Schaftfur Osteo Synthes Fragen (AO/ASIF) in 1996 designed a new medullary device, the proximal femoral nail (PFN), ${ }^{5}$ this is also a collapsible, centramedullary and biomechanically sounder device with added rotational stability. Several studies show that PFN has its own limitations as in complex unstable intertrochanteric fractures (i.e. fracture of greater trochanter with or without comminution of lateral wall of proximal femur) implant failure is seen in form of non-union, secondary varus collapse, cut-out of proximal screw and reoperation.

Latest implant is proximal femoral locking compression plate (PFLCP) which is currently in clinical use. Several studies show that it has several advantages over DHS and PFN specially in complex comminuted osteoporotic unstable fracture where the lateral wall support has been shattered. ${ }^{6}$ 
So, we planned our study to compare the outcomes of DHS, PFN and PFLCP and to explore possible short comings of these modalities specially PFLCP in case of complex comminuted unstable intertrochanteric fractures in osteoporotic patient. AO classification was used to classify fracture pattern. Results were compared for functional outcome using Harris Hip Score.

\section{METHODS}

This prospective study was conducted at Department of orthopaedics, L.L.R.M. Medical College, Meerut between period of August 2016 to September 2017 on 62 patients who were attended in OPD and emergency. Patients were included in the study as decided by inclusion and exclusion criteria and operative intervention for each group was decided on the basis of radiological examination. Approval was taken from the college ethical committee. During the period the patients who were attended the hospital were enrolled as sample size.

Statistical analysis is done by SPSS 16.0 version software. The following tests and tools were used for the statistical analysis of the observations and results. ANOVA Test is done to compare the means of three groups and POST HOC Analysis/Boneferroni Test is used to find out which group is significantly different from rest of the groups. P value $<0.01$ is considered significant.

\section{Inclusion Criteria}

- All Patients with close intertrochanteric fracture above 18 years.

- $\quad$ Fractures within 2 weeks.

- Patient who Ready to give informed consent for minimum 12 months of follow up.

- No medical contraindication to general anaesthesia or regional anaesthesia.

\section{Exclusion Criteria}

- Pathological fractures.

- Compound fractures.

- Polytrauma patients and patients with co-morbidities (i.e. politic and paralytic limb).

- Patient is on chemotherapy or radiotherapy.

History was taken from each patient regarding mode of injury, time since injury. All patients were examined clinically and investigated thoroughly. A day before surgery the patients were subjected to pre anaesthesia check-ups, surgical site of each patient was prepared, and informed consent were taken from each patient or his/her attendants. Each patient was received a single dose antibiotic half an hour before induction of anaesthesia. I.V antibiotic was given to all patients for 7 days. Pain was relieved by analgesic. Wound dressings were done at 3rd post-operative day in routine or anytime when needed for proper care. Stitches were removed on 10-12th post op day

All patients were followed at an interval of 4 weeks till 3 months and patents were assessed clinically, and radiologically, then at the interval of 3 months for period of 1 year. Analysis of results was done at 1 month, 3 month, 6 month and 12 month as per Harris hip scoring system.

\section{Harris Hip Scoring System}

Maximum Points 100.

1. Pain relief: (44)

2. Function (47)

3. Range of motion (5)

4. Absence of Deformity (4)

\begin{tabular}{|c|c|c|}
\hline S/N & Score & Rating \\
\hline 1. & $90-100$ & Excellent \\
\hline 2. & $80-89$ & Good \\
\hline 3. & $70-79$ & Fair \\
\hline 4. & $<70$ & Poor \\
\hline \multicolumn{3}{|c|}{ Table 1 } \\
\hline
\end{tabular}

\section{RESULTS}

Out of the 62 patients, 34 patients (54.83\%) were male and 28 patients $(45.16 \%)$ female. The age of patients recorded in our series ranged between 20-90 yrs. Mean age for PFN group is 56 years, mean age for DHS group is 58 years, and mean age for PFLCP group is 64 years.

\begin{tabular}{|c|c|c|c|c|c|c|c|c|}
\hline $\begin{array}{c}\text { Mode of } \\
\text { Injury }\end{array}$ & \multicolumn{2}{|c|}{ PFN } & \multicolumn{2}{|c|}{ DHS } & \multicolumn{2}{c|}{ PFLCP } & Total & $\begin{array}{c}\text { Total } \\
\%\end{array}$ \\
\hline RTA & 06 & $30 \%$ & 12 & $37.5 \%$ & 04 & $40 \%$ & 22 & 35.8 \\
\hline Domestic fall & 13 & $65 \%$ & 18 & $56.2 \%$ & 06 & $60 \%$ & 37 & 60.4 \\
\hline Assault & 01 & $5 \%$ & 02 & $6.25 \%$ & 0 & $0 \%$ & 03 & 3.75 \\
\hline \multicolumn{6}{c|}{ Table 2. Mode of Injury } \\
\hline
\end{tabular}

We found that intertrochanteric fracture due to domestic fall (60.4\%) was most common mode of injury, followed by fracture due to road traffic injury (35.83\%). $60 \%$ fractures occurred on left side and $40 \%$ on the right side.

\begin{tabular}{|c|c|c|c|}
\hline AO Classification & PFN & DHS & PFLCP \\
\hline A1 & 04 & 02 & 01 \\
\hline A2 & 05 & 12 & 01 \\
\hline A3 & 11 & 18 & 08 \\
\hline \multicolumn{3}{|c|}{ Table 3. Classification of Fractures } \\
\hline
\end{tabular}

\begin{tabular}{|c|c|c|c|c|c|c|}
\hline Type & \multicolumn{3}{|c|}{ PFN } & \multicolumn{2}{c|}{ DHS } & \multicolumn{2}{c|}{ PFLCP } \\
\hline Stable (25) & 09 & $45 \%$ & 14 & $43.75 \%$ & 02 & $20 \%$ \\
\hline $\begin{array}{c}\text { Unstable } \\
(37)\end{array}$ & 11 & $55 \%$ & 18 & $56.25 \%$ & 08 & $80 \%$ \\
\hline \multicolumn{5}{|c|}{ Table 4. Fracture Pattern } \\
\hline
\end{tabular}

According to AO classification 25 patients (40.3\%) were stable and 37 patients (59.6\%) were unstable. In our study we got most of patients with A3 type unstable fractures. In PFN group 11 patients (55\%), in DHS group 18 patients (56.26) and in PFLCP group 8 patients (80) were with A3 type fractures. While patients with A1 type fractures were least in numbers. In PFN group 4 patients (20\%), in DHS group 2 patients $(6.25 \%)$ and in PFLCP group 1 patient (10\%) were with A1 type fractures.

In our study shortest operation time was in DHS group with overall mean of 46.5 min followed by PFN ( 60 minutes) and PFLCP (75 minutes) shows significant difference in operation time PFN vs. DHS (P- value 0.001) PFN vs. PFLCP (P-value 0.000 ) and DHS vs. PFLCP (P-value 0.000).

In our study patients with stable fracture mobilizes early as compare to unstable fracture. In PFN group overall mean for mobilization is about 9.75 days. While for DHS group it is 
about 12.7 days but in PFLCP group patients restrict to weight-bearing for about 19.6 days.

Patient treated with PFN was having least duration of hospital stay average of about 12 days, ranging from 10-14 days while patients treated with DHS was having average stay of 13 days ranging from 11-15 days. After intervention in PFLCP group average duration of hospital stay was 15 days ranging from 12-18 days postoperatively.

\begin{tabular}{|c|c|c|c|c|c|c|c|c|c|}
\hline \multirow{2}{*}{$\begin{array}{c}\text { Group and } \\
\text { Classification }\end{array}$} & \multirow{2}{*}{ No. } & \multicolumn{2}{|c|}{$\begin{array}{c}01 \\
\text { Month }\end{array}$} & \multicolumn{2}{|c|}{$\begin{array}{c}03 \\
\text { Month } \\
\end{array}$} & \multicolumn{2}{|c|}{$\begin{array}{c}06 \\
\text { Month }\end{array}$} & \multicolumn{2}{|c|}{$\begin{array}{c}12 \\
\text { Month }\end{array}$} \\
\hline & & Mean & S/D & Mean & S/D & Mean & S/D & Mean & S/D \\
\hline PFN (A) & 20 & 44.3 & \multirow{3}{*}{8.5} & \begin{tabular}{|l|}
72.0 \\
\end{tabular} & \multirow{3}{*}{ \pm 10.8} & 88.3 & \multirow{3}{*}{ \pm 4.9} & \begin{tabular}{|l|}
94.8 \\
\end{tabular} & \multirow{3}{*}{ \pm 3.6} \\
\hline Stable & 09 & 47.3 & & 74.5 & & 89.4 & & 85.4 & \\
\hline Unstable & 11 & 41.9 & & 69.5 & & 69.5 & & 93.6 & \\
\hline DHS ( & 3 & 37.9 & \multirow{3}{*}{ \pm 5.9} & 60.4 & \multirow{3}{*}{ \pm 9.2} & 81.7 & \multirow{3}{*}{ \pm 6.6} & 91.8 & \multirow{3}{*}{ \pm 6.5} \\
\hline $\mathrm{b}$ & 14 & 39.2 & & 62.8 & & 84 & & 93.2 & \\
\hline Unstable & 18 & 36.9 & & 58.8 & & 80 & & \begin{tabular}{|l|}
88.2 \\
\end{tabular} & \\
\hline PFLC & 10 & 35.8 & \multirow{3}{*}{5.9} & 52.9 & \multirow{3}{*}{ \pm 7.4} & 76.1 & \multirow{3}{*}{ \pm 8.3} & 89.3 & \multirow{3}{*}{ \pm 8.5} \\
\hline & 2 & 47.8 & & 55.4 & & 76.5 & & 89.6 & \\
\hline Unstable & 8 & 35.3 & & 50.4 & & 76.0 & & 89.0 & \\
\hline \multicolumn{10}{|c|}{ able 5. Evaluation of Harris Hip Score } \\
\hline
\end{tabular}

In present study at 01 month mean score for (PFN 44.3 vs. DHS 37.9) $\mathrm{p}=0.002$, PFN 44.3 vs. PFLCP 37.8 ) $\mathrm{p}=0.002$ shows significant improvement in PFN group. At 3 months mean score for (PFN 72 vs. DHS 60.4) $p=0.00$, (PFN 72 vs. PFLCP 52.9) $\mathrm{p}=0.000$ shows significant improvement in PFN group. At 6 months, mean score for (PFN 88.3 vs. DHS 81.7) $\mathrm{p}=0.000$, (PFN 88.3 vs. PFLCP 76.1 ) $\mathrm{p}=0.000$ shows highly significant improvement in PFN group. But at 1 year of follow up Harris Hip score for PFN $=94.8$ vs. DHS $=91$ vs. PFLC $=$ 89.1 shows insignificant difference in functional status of patients belong to either group.

\begin{tabular}{|c|c|c|c|c|c|c|}
\hline \multicolumn{2}{|c|}{ ANOVA Test } & \multirow{2}{*}{\begin{tabular}{|c|}
$\begin{array}{c}\text { Sum of } \\
\text { Squares }\end{array}$ \\
681.0
\end{tabular}} & \multirow{2}{*}{\begin{tabular}{|c|}
$\begin{array}{c}\text { Degree of } \\
\text { Freedom }\end{array}$ \\
2 \\
\end{tabular}} & \multirow{2}{*}{\begin{tabular}{|c|}
$\begin{array}{c}\text { Mean } \\
\text { Square }\end{array}$ \\
340.5 \\
\end{tabular}} & $\begin{array}{c}\text { F- } \\
\text { Stats }\end{array}$ & \begin{tabular}{|c|}
$p$ \\
Value
\end{tabular} \\
\hline \multirow{3}{*}{$\begin{array}{l}\text { After 1 } \\
\text { Month }\end{array}$} & $\begin{array}{l}\text { Between } \\
\text { Groups }\end{array}$ & & & & \multirow{3}{*}{6.9} & \multirow{3}{*}{.002} \\
\hline & Within Groups & 2911.0 & 59 & 49.3 & & \\
\hline & Total & 3592.2 & 61 & & & \\
\hline
\end{tabular}

\section{Comparison Within the Groups}

ANOVA test gives $p$ value of 0.02 indicating there is significant improvement in functional outcomes of patients in all 3 groups at 01 month of follow up.

\begin{tabular}{|c|c|c|c|c|c|c|c|}
\hline \multirow{2}{*}{$\begin{array}{l}\text { Dependent } \\
\text { Variable }\end{array}$} & \multirow{2}{*}{$\begin{array}{c}\text { (I) } \\
\text { Group }\end{array}$} & \multirow{2}{*}{$\begin{array}{c}\text { (J) } \\
\text { Group }\end{array}$} & \multirow{2}{*}{$\begin{array}{c}\text { Mean } \\
\text { Difference } \\
(I-J)\end{array}$} & \multirow{2}{*}{$\begin{array}{l}\text { Std. } \\
\text { Error }\end{array}$} & \multirow{2}{*}{$\begin{array}{c}\text { p } \\
\text { Value }\end{array}$} & \multicolumn{2}{|c|}{$\begin{array}{c}95 \% \\
\text { Confidence } \\
\text { Interval }\end{array}$} \\
\hline & & & & & & \begin{tabular}{|l|} 
Lower \\
Bound
\end{tabular} & $\begin{array}{l}\text { Upper } \\
\text { Bound }\end{array}$ \\
\hline \multirow{6}{*}{$\begin{array}{l}\text { After } 1 \\
\text { Month }\end{array}$} & \multirow{2}{*}{$\begin{array}{c}\text { Group } \\
\text { A }\end{array}$} & $\begin{array}{c}\text { Group } \\
\text { B }\end{array}$ & 8.49 & 2.72 & .008 & 1.8 & 15.2 \\
\hline & & Group C & 6.43 & 2.00 & .006 & 1.5 & 11.4 \\
\hline & \multirow{2}{*}{$\underset{B}{G r o u p}$} & \begin{tabular}{|c|} 
Group \\
A
\end{tabular} & -8.49 & 2.72 & .008 & -15.2 & -1.80 \\
\hline & & Group C & -2.06 & 2.54 & 1.000 & -8.3 & 4.2 \\
\hline & \multirow{2}{*}{$\begin{array}{c}\text { Group } \\
\text { C }\end{array}$} & \begin{tabular}{c|} 
Group \\
A
\end{tabular} & -6.43 & 2.00 & .006 & -11.4 & -1.5 \\
\hline & & $\begin{array}{c}\text { Group } \\
\text { B }\end{array}$ & 2.06 & 2.54 & 1.000 & -4.2 & 8.3 \\
\hline \multicolumn{8}{|c|}{$\begin{array}{l}\text { Table 7. After } 01 \text { Month of Follow Up: Post Hoc Analysis } \\
\text { (Bonferroni Test)/Multiple Comparison }\end{array}$} \\
\hline
\end{tabular}

\section{Comparison Among the Groups}

For comparison we applied Bonferroni/ post HOC analysis $p$ value for PFN vs. DHS is $0.008(<0.05)$ and $p$ value for PFN vs. PFLCP is $0.006(<0.05)$ indicating a significant improvement in functional score of patients in PFN group as compared to DHS and PFLCP group. While p-value for DHS vs. PFLCP is $1.00(>0.05)$ shows insignificant difference in functional status of patients in these groups.

\begin{tabular}{|c|c|c|c|c|c|c|}
\hline \multicolumn{2}{|c|}{ ANOVA Test } & $\begin{array}{c}\text { Sum of } \\
\text { Squares }\end{array}$ & $\begin{array}{c}\text { Degree of } \\
\text { Freedom }\end{array}$ & $\begin{array}{c}\text { Mean } \\
\text { Square }\end{array}$ & $\begin{array}{c}\text { F- } \\
\text { Stats }\end{array}$ & $\begin{array}{c}\text { V } \\
\text { Value. }\end{array}$ \\
\hline \multirow{4}{*}{$\begin{array}{c}\text { After 03 } \\
\text { Months }\end{array}$} & $\begin{array}{c}\text { Between } \\
\text { Groups }\end{array}$ & 2837.8 & 2 & 1418.9 & & \\
\cline { 2 - 5 } & $\begin{array}{c}\text { Within } \\
\text { Groups }\end{array}$ & 5677.6 & 59 & 96.2 & & \\
\cline { 2 - 5 } & Total & $\mathbf{8 5 1 5 . 4}$ & $\mathbf{6 1}$ & & & \\
\hline \multicolumn{6}{|c|}{ Table 8. After 3 Months of Follow Up } \\
\hline
\end{tabular}

\section{Comparison Within the Groups}

ANOVA test gives p value of 0.000 in all 3 groups indicating highly significant improvement in functional score of patients in all groups at 03 month of follow up.

\begin{tabular}{|c|c|c|c|c|c|c|c|}
\hline \multirow{2}{*}{$\begin{array}{c}\text { Dependent } \\
\text { Variable }\end{array}$} & \multirow{2}{*}{$\begin{array}{c}\text { (I) } \\
\text { Group }\end{array}$} & \multirow{2}{*}{$\begin{array}{c}\text { (J) } \\
\text { Group }\end{array}$} & \multirow{2}{*}{$\begin{array}{c}\text { Mean } \\
\text { Difference } \\
\text { (I-J) }\end{array}$} & \multirow{2}{*}{$\begin{array}{c}\text { Std. } \\
\text { Error }\end{array}$} & \multirow{2}{*}{$\begin{array}{c}\mathbf{p} \\
\text { Value }\end{array}$} & \multicolumn{2}{|c|}{$\begin{array}{c}95 \% \\
\text { Confidence } \\
\text { Interval }\end{array}$} \\
\hline & & & & & & $\begin{array}{l}\text { Lower } \\
\text { Bound }\end{array}$ & $\begin{array}{l}\text { Upper } \\
\text { Bound }\end{array}$ \\
\hline \multirow{6}{*}{$\begin{array}{l}\text { After } 3 \\
\text { Months }\end{array}$} & \multirow{2}{*}{$\begin{array}{c}\text { Group } \\
\text { A }\end{array}$} & Group B & 19.05 & 3.80 & .000 & 9.7 & 28.4 \\
\hline & & Group C & 11.55 & 2.80 & \begin{tabular}{|l|}
.000 \\
\end{tabular} & 4.7 & 18.4 \\
\hline & \multirow{2}{*}{ Group B } & Group A & -19.05 & \begin{tabular}{|l|}
3.80 \\
\end{tabular} & \begin{tabular}{|l|l|}
.000 \\
\end{tabular} & \begin{tabular}{|l|}
-28.4 \\
\end{tabular} & -9.7 \\
\hline & & Group C & -7.50 & 3.55 & \begin{tabular}{|l}
.117 \\
\end{tabular} & \begin{tabular}{|l|}
-16.3 \\
\end{tabular} & 1.3 \\
\hline & \multirow{2}{*}{ Group C } & Group A & -11.55 & 2.80 & \begin{tabular}{|l|}
.000 \\
\end{tabular} & -18.4 & -4.7 \\
\hline & & Group B & 7.50 & 3.55 & .117 & -1.3 & 16.3 \\
\hline
\end{tabular}

Table 9. After 3 Months of Follow Up: Post Hoc Analysis (Bonferroni Test)/Multiple Comparison

\section{Comparison Among the Groups}

$\mathrm{p}$ value for PFN vs. DHS and PFN vs. PFLCP is 0.00 while for DHS vs. PFLCP $p$ value is $0.117(>0.05)$. Indicating PFN group patient had better functional score at 3 months than DHS and PFLCP group it is because of early mobilisation and weight bearing in this group. But there is insignificant difference in functional score of DHS and PFLCP group at 3 months of follow up.

\begin{tabular}{|c|c|c|c|c|c|c|}
\hline \multicolumn{2}{|c|}{ ANOVA Test } & $\begin{array}{c}\text { Sum of } \\
\text { Squares }\end{array}$ & $\begin{array}{c}\text { Degree of } \\
\text { Freedom }\end{array}$ & $\begin{array}{c}\text { Mean } \\
\text { Square }\end{array}$ & $\begin{array}{c}\text { F- } \\
\text { Stats }\end{array}$ & $\begin{array}{c}\text { p } \\
\text { Value }\end{array}$ \\
\hline \multirow{2}{*}{$\begin{array}{c}\text { After } \\
\text { 06 } \\
\text { Months }\end{array}$} & $\begin{array}{c}\text { Between } \\
\text { Groups }\end{array}$ & 1102.2 & 2 & 551.1 & & \\
\cline { 2 - 5 } & $\begin{array}{c}\text { Within } \\
\text { Groups }\end{array}$ & 2568.2 & 59 & 43.5 & 12.7 & .000 \\
\cline { 2 - 5 } & \multicolumn{7}{|c|}{ Total } & $\mathbf{3 6 7 0 . 4}$ & $\mathbf{6 1}$ & & & \\
\hline \multicolumn{7}{|c|}{ Table 10. After 6 Months of Follow Up } \\
\end{tabular}

\section{Comparison Within the Groups}

p-value is 0.00 for all 3 group it inferences a highly significant improvement in functional score of patients at 6 months of follow up. 


\begin{tabular}{|c|c|c|c|c|c|c|c|}
\hline \multirow{2}{*}{$\begin{array}{l}\text { Dependent } \\
\text { Variable }\end{array}$} & \multirow{2}{*}{ Group } & \multirow{2}{*}{$\begin{array}{l}\text { (J) } \\
\text { Group }\end{array}$} & \multirow{2}{*}{\begin{tabular}{|} 
Mean \\
Difference \\
(I-J)
\end{tabular}} & \multirow{2}{*}{$\begin{array}{l}\text { Std. } \\
\text { Error }\end{array}$} & \multirow{2}{*}{$\begin{array}{c}\text { p- } \\
\text { Value }\end{array}$} & \multicolumn{2}{|c|}{$\begin{array}{c}95 \% \\
\text { Confidence } \\
\text { Interval }\end{array}$} \\
\hline & & & & & & \begin{tabular}{l|} 
Lower \\
Bound
\end{tabular} & $\begin{array}{l}\text { Upper } \\
\text { Bound }\end{array}$ \\
\hline \multirow{6}{*}{$\begin{array}{l}\text { After } 6 \\
\text { Months }\end{array}$} & Group & Group B & 12.28 & 2.56 & .003 & 6.0 & 18.6 \\
\hline & A & Group C & 6.60 & 1.88 & .000 & 2.0 & 11.2 \\
\hline & Group & Group A & -12.28 & 2.56 & .000 & -18.6 & -6.0 \\
\hline & B & Group C & -5.67 & 2.39 & .063 & \begin{tabular}{|l|}
-11.6 \\
\end{tabular} & .2 \\
\hline & Group & Group A & -6.60 & \begin{tabular}{|l|}
1.88 \\
\end{tabular} & .003 & \begin{tabular}{|l|}
-11.2 \\
\end{tabular} & -2.0 \\
\hline & C & Group B & 5.67 & 2.39 & .063 & -.2 & 11.6 \\
\hline \multicolumn{8}{|c|}{$\begin{array}{l}\text { Table 11. After } 6 \text { Months of Follow Up: Post Hoc Analysis } \\
\text { (Bonferroni Test)/Multiple Comparison }\end{array}$} \\
\hline
\end{tabular}

\section{Comparison Among the Groups}

$\mathrm{p}$ value for PFN vs. DHS is $0.003(<0.05), \mathrm{p}$ value for PFN vs. PFLCP is 000 , it inferences that PFN group had better outcome than DHS and PFLCP group followed by DHS group while $\mathrm{p}$ value for DHS vs. PFLCP is 0.063 indicating insignificant difference between these two groups.

\begin{tabular}{|c|c|c|c|c|c|c|}
\hline \multicolumn{2}{|c|}{ ANOVA Test } & $\begin{array}{c}\text { Sum of } \\
\text { Squares }\end{array}$ & $\begin{array}{c}\text { Degree of } \\
\text { Freedom }\end{array}$ & $\begin{array}{c}\text { Mean } \\
\text { Square }\end{array}$ & $\begin{array}{c}\text { F- } \\
\text { Stats }\end{array}$ & $\begin{array}{c}\text { V } \\
\text { Value }\end{array}$ \\
\hline \multirow{2}{*}{$\begin{array}{c}\text { After } \\
12\end{array}$} & $\begin{array}{c}\text { Between } \\
\text { Groups }\end{array}$ & 268.0 & 2 & 134.0 & & \\
\cline { 2 - 5 } Months & $\begin{array}{c}\text { Within } \\
\text { Groups }\end{array}$ & 2346.5 & 59 & 39.8 & \multirow{2}{*}{3.4} & .41 \\
\cline { 2 - 5 } & \multicolumn{7}{|c|}{ Total } & 2614.5 & 61 & & & \\
\hline \multicolumn{7}{|c|}{ Table 12. After 12 Months of Follow Up } \\
\hline
\end{tabular}

\section{Comparison Within the Groups}

$p$ value is 0.041 showing significant increment in functional score of patients in all groups.

\begin{tabular}{|c|c|c|c|c|c|c|c|}
\hline \multirow{2}{*}{$\begin{array}{l}\text { Dependent } \\
\text { Variable }\end{array}$} & \multirow{2}{*}{ (I) } & \multirow{2}{*}{$\begin{array}{l}\text { (J) } \\
\text { Group }\end{array}$} & \multirow{2}{*}{$\begin{array}{c}\text { Mean } \\
\text { Difference } \\
\text { (I-J) }\end{array}$} & \multirow{2}{*}{$\begin{array}{c}\text { Std. } \\
\text { Error }\end{array}$} & \multirow{2}{*}{$\begin{array}{c}\text { p- } \\
\text { Value }\end{array}$} & \multicolumn{2}{|c|}{$\begin{array}{c}95 \% \\
\text { Confidence } \\
\text { Interval }\end{array}$} \\
\hline & & & & & & $\begin{array}{l}\text { Lower } \\
\text { Bound }\end{array}$ & $\begin{array}{l}\text { Upper } \\
\text { Bound }\end{array}$ \\
\hline \multirow{6}{*}{$\begin{array}{l}\text { After } 12 \\
\text { Months }\end{array}$} & Group & Group B & 5.65 & \begin{tabular}{|l|}
2.44 \\
\end{tabular} & .121 & -.4 & 11.7 \\
\hline & A & Group C & 3.77 & 1.80 & \begin{tabular}{|l|l|}
.073 \\
\end{tabular} & -.7 & 8.2 \\
\hline & Group & Group A & -5.65 & 2.44 & .073 & -117 & .4 \\
\hline & B & Group C & -1.88 & 2.28 & 1.000 & -7.5 & 3.8 \\
\hline & Group & Group A & $\begin{array}{l}-3.77 \\
\end{array}$ & \begin{tabular}{|l|}
1.80 \\
\end{tabular} & .121 & -8.2 & .7 \\
\hline & $\mathrm{C}$ & Group B & 1.88 & \begin{tabular}{|l|}
2.88 \\
\end{tabular} & 1.000 & -3.8 & 7.5 \\
\hline \multicolumn{8}{|c|}{$\begin{array}{l}\text { Table 13. After } 12 \text { Months of Follow Up: Post Hoc Analysis } \\
\text { (Bonferroni Test)/Multiple Comparison }\end{array}$} \\
\hline
\end{tabular}

\section{Comparison Among the Groups}

At the end of 12 months of follow p value for PFN vs. DHS group is $0.12, p$ value for PFN vs. PFLCP is $0.073(>0.05)$ and $\mathrm{p}$ value for DHS vs. PFLCP is 1.00 all $\mathrm{p}$ values are not significant shows insignificant difference in functional outcome of patients. It inferences that most of patients achieved approximately same level of functional outcome at the end of one years.

\begin{tabular}{|c|c|c|c|c|c|c|}
\hline Groups & \multicolumn{2}{|c|}{ Patients } & Results & Percent & $\begin{array}{l}\text { Mean } \\
\text { Score }\end{array}$ & S/D \\
\hline \multirow[b]{2}{*}{ PFN (A) } & \multirow[b]{2}{*}{20} & 16 & Excellent & $80.0 \%$ & \multirow[b]{2}{*}{94.8} & \multirow[b]{2}{*}{ \pm 3.6} \\
\hline & & 04 & Good & $20.0 \%$ & & \\
\hline \multirow{3}{*}{ DHS (B) } & \multirow{3}{*}{32} & 21 & Excellent & $65.6 \%$ & \multirow{3}{*}{90.8} & \multirow{3}{*}{ \pm 6.5} \\
\hline & & 09 & Good & $28.1 \%$ & & \\
\hline & & 02 & Poor & $6.3 \%$ & & \\
\hline \multirow{3}{*}{ PFLCP (C) } & \multirow{3}{*}{10} & 06 & Excellent & $60 \%$ & \multirow{3}{*}{89.3} & \multirow{3}{*}{ \pm 8.5} \\
\hline & & 03 & Good & $30 \%$ & & \\
\hline & & 01 & Poor & $10 \%$ & & \\
\hline \multicolumn{7}{|c|}{ Table 12. Functional Score and Result at 1 Year } \\
\hline
\end{tabular}

In present study, we used Harris Hip Score for comparison. At 3 months mean score for (PFN = 72 vs. DHS = 60.4) $\mathrm{p}=0.00,(\mathrm{PFN}=72$ vs. $\mathrm{PFLCP}=52.9) \mathrm{p}=0.000$ shows significant improvement in PFN group. But at 1 year of follow up Harris Hip score for PFN $=94.8$ vs. DHS $=91$ vs. PFLC $=$ 89.1 shows insignificant difference in functional status of patients belong to either group.

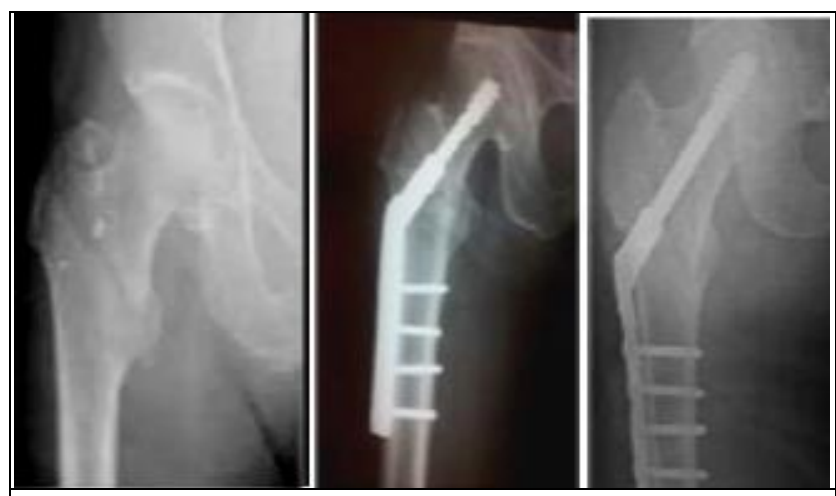

Figure 1. Intertrochanteric Fracture Managed by DHS

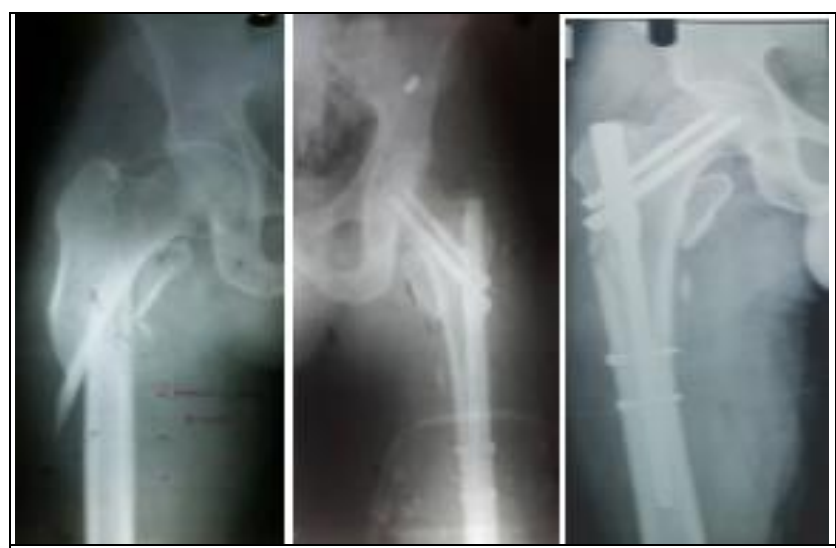

Figure 2. Intertrochanteric Fracture Managed by PFN

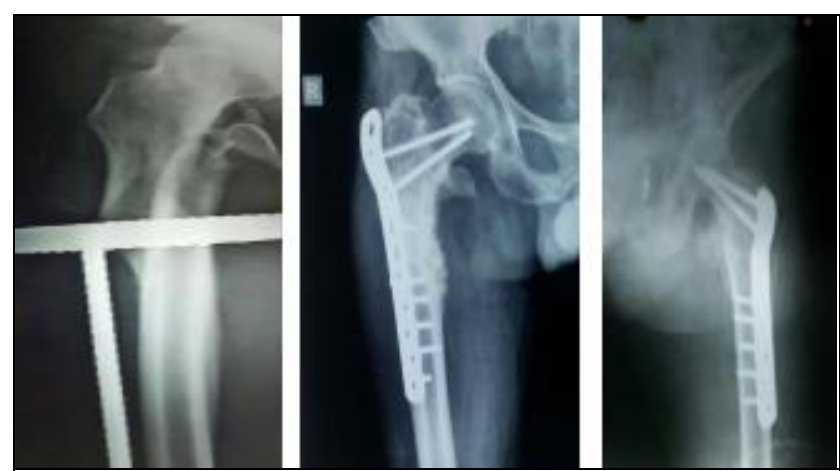

Figure 3. Intertrochanteric Fracture Managed by PFLCP 


\section{DISCUSSION}

Closed or open reduction and internal fixation for trochanteric fracture is the treatment of choice now a day. Conservative treatment for these fracture are now matter of past and not even a thought except in extreme cases of morbidity. In spite of ample number of studies, the debate is still continued for best internal fixation device for trochanteric fractures, especially complex comminuted type unstable fractures.

Most of patients in our study were from 5 th to 7 th decade, Mean age for PFN 56 years, Mean age for DHS 58 years while for PFLCP 64 years. Cleveland et $\mathrm{al}^{7}$ pointed out higher incidence of intertrochanteric fractures in elderly people because of poor vision, senile osteoporosis, and low muscle mass to absorb energy of trauma. Ahrengart L, Tornkvist H, Fornander $\mathrm{P}$ et al $^{8}$ reported average age 80 years for male and 78 year for female. Tyllionksi $\mathrm{M}$ et al $^{9}$ reported average age 71.3 years. In our study overall average age is 58.32 years which is lower in comparison to above mentioned studies probably because of lower life expectancy, early osteoporosis and poor built in Asian population specially of developing countries like India.

Harrington and Johnton ${ }^{10}$ reported $42 \%$ male and 58\% female, G.S.Kulkarni ${ }^{11}$ in 1984 concluded average age of 62 years. Kudema et $\mathrm{al}^{12} 36 \%$ male and $64 \%$ female, Poihgen first and Schnable 13 17\% male and $83 \%$ female. In our study, male and female ratio 1.2:1(34 male 28 female) which is in contrast to most of the reported literature. In this part of India, females are mostly confined to their houses in this age group.

It is very difficult to classify the mode of injury accurately. We divided the mode of injury into 3 broad categories as RTA, domestic fall and assault on the basis of this classification maximum cases were observed in domestic fall i.e. 37 patients (59.67\%) out of 62 . According to Cummings and Nevit,14 there is inadequate protective reflexes to reduce energy of fall, inadequate local shock absorbers and none strength in elderly patient. Keneth J. Koval and Joseph D. Zuckerman ${ }^{15}$ observed that $90 \%$ of hip fractures in the elderly result from a simple fall.

In our study average hospital stay of patient in PFN group was about 12 days ranging from 10-14 days, in DHS group it was 13 days ranging from 11-15 days and in PFLCP group 15 days ranging from 12 to 18 days. Shishir Murugharaj et al16 reported average hospital stay of 7.9 days in patients treated with PFN and 12.04 days in patients treated with DHS.

In present study we used AO classification to classify the fracture pattern. In PFN group 9 cases out of 20, in DHS 14 out of 32 cases and in PFLCP 2 out of 10 cases was having stable fracture configuration. In present study unstable fractures accounts for 37 (59.67\%) out of 62 patients while stable fracture accounts for $40.3 \%$ cases of total 62 patients. Wolfgang et $\mathrm{al}^{17}$ reported $79 \%$ stable and $21 \%$ unstable type fracture, Neilson, B.P. et al ${ }^{18}$ reported $28 \%$ stable and $72 \%$ unstable trochanteric fracture, Larsson S, Friberg $\mathrm{S}$ et al ${ }^{19}$ reported a series of $35 \%$ stable and $65 \%$ unstable trochanteric fracture.

In the present study the patients were mobilized in bed to a sitting position the day after surgery to decrease the incidence of pulmonary thromboembolism and urinary tract complication. Gentle hip and knee exercise in the bed was promoted in all groups. A Frame/walker walking program with partial weight bearing was allowed after 1 postoperative week for PFN and DHS groups. The ability to resume ambulation after trochanteric fracture is related to several factors besides simple fracture healing. NJ. Little, V. Verma. et al ${ }^{20}$ suggested the reason for this to be post-operative factors: pain, muscle dysfunction and medical co-morbidities.

In our study mean operative time for PFN group is 60 minutes and for DHS in 46.5 minutes while for PFLCP group is 75 minutes. This is in accordance with previous studies Wie Ting lee et $\mathrm{al}^{21}$ reported mean operating time for PFLCP on 13 cases with multi-fragmentary unstable intertrochanteric fracture was 151.3 minutes while for stable fractures 116 minutes. Nayer asif et al ${ }^{22}$ reported average operating time in 27 patients operated with PFLCP was about 75 minutes.

In present study we used Harris Hip Score for comparison. At 3 months mean score for $(\mathrm{PFN}=72 \mathrm{vs}$. DHS = 60.4) $\mathrm{p}=0.00$, (PFN=72 vs. PFLCP $=52.9) \mathrm{p}=0.000$ shows significant improvement in PFN group. But at 1 year of follow up Harris Hip score for PFN $=94.8$ vs. DHS $=91$ vs. PFLC $=$ 89.1 shows insignificant difference in functional status of patients belong to either group.

In PFN group after 1 year of follow up score of 16 patients was above 90 irrespective of fracture pattern, 1 patient had non-union with Harris Hip Score of 86.6 which is lowest in PFN group in our study. We had excellent results with PFN in $80 \%$ of patients.

In DHS group after 1 year follow up we had mean function score of 91.0. Two patients, who had comminuted unstable complex fracture, had poor functional outcome with score of 68.5 and 69.8 respectively. In our study $65.625 \%$ patients in DHS group had excellent functional outcomes.

In PFLCP group at 1 year of follow up 1 patient developed implant failure with Harris Hip Score of 65.7 which is lowest in this group, while the mean functional score for PFLCP group after 1 year is 89.1 . In our study $60 \%$ patient in this group had excellent functional outcomes.

Domingo L J, et $\mathrm{al}^{23}$ followed prospectively 295 patients with trochanteric fractures treated with the PFN. Clinical and radiographic controls were performed at 1, 3 and 6 months. They showed that previous walking ability was recovered by 71\%. Wei Ting Lee et al21 reported mean Harris Hip Score 80.1 for stable and 69.1 for unstable multi-fragmentary fractures treated with PFLCP. Govindasamy et al ${ }^{6}$ reported mean Harris Hip Score 85.5 (83-94) with no poor result in 18 patients treated with PFLCP. Mohsen Mardani et al ${ }^{24}$ reported Harris Hip Score 24 patients treated with DHS with stable fracture was 87.08 and for unstable fracture (36 patients) score was 84.61 , while score for patients treated with PFLCP having stable fractures was 85.43 and with unstable fracture was 81.20.

\section{CONCLUSIONS}

PFN had good to excellent outcomes. Only one patient developed non-union, but he still walks with slight limping. So PFN offers high rotational stability, compression at fracture site, create a shorter lever arm, so had decreased rate of mechanical failure, reduced hospital stay, early mobilization, early rehabilitation and faster union as compared to DHS and PFLCP.

DHS offers advantages over PFN and PFLCP in terms of lesser operation time, lesser cost of implant, lesser radiation 
exposure and requiring least surgical expertise. But it should be used cautiously in unstable comminuted intertrochanteric fractures as it leads to varus collapse in $12.5 \%$ cases.

Proximal femoral locking plate also seems to be a feasible alternative to PFN and DHS in certain complex comminuted unstable osteoporotic intertrochanteric fractures as it locks the fracture in reduced position achieved by surgeon without controlled collapse. There was varus collapsing in one patient. One of major drawbacks of using PFLCP is that weight bearing has to be controlled and allowed only after radiological evidence of fracture healing.

\section{REFERENCES}

[1] Kumar A, Rao BS, Bhaskar SK. Long term functional outcomes of intertrochanteric femur fractures treated with screw slide plate and proximal femur nail: Retrospective study. International Journal of Orthopaedic Science 2018;4(2):672-6.

[2] Dimon JH, Hughston JC. Unstable intertrochanteric fractures of the hip. J Bone Joint Surg Am 1967;49(3):440-50.

[3] Gulberg B, Johnell 0, Kanis JA. World-wide projections for hip fractures. Osteoporos Int 1997;7(5):407-13.

[4] Evans EM. The treatment of trochanteric fractures of the femur. J Bone Joint Surg Br 1949;31B(2):190-203.

[5] Canale TS, Beaty JH. Campbell's Operative orthopaedics. $11^{\text {th }}$ edn. Elsevier 2008: p. 3239.

[6] Govindasamy R, Gnanasundaram R, Kasirajan S, et al. Proximal femur locking compression plate in complex proximal femoral fractures: a retrospective analysis. 2016;2(3):104-8.

[7] Cleveland M, Bosworth DM, Thompson FR, et al. A ten year analysis of intertrochanteric fractures of the femur. J Bone Joint Surg 1959;41-A:1399-408.

[8] Ahrengart L, Tornkvist $\mathrm{H}$, Fornander $\mathrm{P}$, et al. A randomized study of the compression hip screw and Gamma nail in 426 fractures. Clinical Orthopaedics and Related Research 2002;(401):209-22.

[9] Tyllianakis M, Panagopoulos A, Papadopoulos A. et al. Treatment of extracpsular hip fractures with the proximal femoral nail: long term results in 45 patients. Acta Orthop Belg 2004;70(5):444-54.

[10] Harrington P, Nihal A, Singhania AK, et al. Intramedullary hip screw versus sliding hip screw for unstable intertrochanteric femoral fractures in the elderly. Injury 2002;33(1):23-8.

[11] Kulkarni GS, Limaye R, Kulkarni M, et al. Treatment of trochanteric fractures of the hip by modified Richard's compressing and collapsing screw. Indian Journal of Orthopaedics 1984;18(1):30-4.
[12] Kuderna H, Bohler N, Collon DJ. Treatment of intertrochanteric and subtrochanteric fractures of the hip by Ender method. J Bone Joint Surg 1976;58(5):604-11.

[13] Poigenfurst J, Schnabl P. Multiple intramedullary nailing of pertrochanteric fractures with elastic nails: operative procedure and results. Injury 1977;9(2):102-13.

[14] Cumming SR, Nevitt MC, Browner WS, et al. Risk factors for hip fracture in white women. Study of Osteoporotic Fractures Research Group. N Eng J Med 1995;332(12):767-73.

[15] Koval KJ, Zuckerman JD. Intertrochanteric fractures. Chapter - 39. In: Buckholz RW, Heckman JD, eds. Rockwood and Green's Fracture in adults. Vol. 2. 5th edn. Philadelphia: Lippincott Williams \& Wilkins 2001: p. 1635-63.

[16] Suranigi SM, Shetty N, Shah HM, et al. Study comparing the advantage of PFN over DHS in patients with subtrochantric fractures. J Medical Thesis 2014;2(1):35-8.

[17] Wolfgang GL, Bryant MH, O'Neill JP. Treatment of intertrochanteric fracture of the femur using sliding screw plate fixation. Clin Orthop Relat Res 1982;(163):148-58.

[18] Nielsen BP, Jelnes R, Rasmussen LB, et al. Trochanteric fractures treated by the McLaughlin nail and plate. Injury (IJCI) 1985;16(5):333-6.

[19] Larsson S, Friberg S, Hansson LI. Trochanteric fractures: influence of reduction and implant position on impaction and complications. Clin Orthop Relat Res 1990;(259):130-9.

[20] Little NJ, Verma V, Fernando C, et al. A prospective trial comparing the Holland nail with the dynamic hip screw in the treatment of intertrochanteric fractures of the hip. J Bone Joint Surg Br 2008;90(8):1073-8.

[21] Lee WT, Murphy D, Kagda FH, et al. Proximal femoral locking compression plate for proximal femoral fractures. J Orthop Surg (Hong Kong) 2014;22(3):28793.

[22] Asif N. Unstable IT fractures fixation - is proximal femoral locked compression plate better than dynamic hip screw. J Orthop 2012;20(6):350-2.

[23] Domingo LJ, Cecilia D, Herrera A, et al. Trochanteric fractures treated with a proximal femoral nail. Int Orthop 2001;25(5):298-301.

[24] Mardani-Kivi M, Mirbolook A, Jahromim KS, et al. Fixation of intertrochanteric fractures: dynamic hip screw versus locking compression plate. Trauma Mon 2013;18(2):67-70. 